\title{
PROBABILIDAD E INCERTIDUMBRE, UNA APROXIMACIÓN DESDE LA HISTORIA DEL PENSAMIENTO
}

Mario Garcia Molina*

$7 \mathrm{n}$ su tesis doctoral, publicada con el título Riesgo, incertidumbre Ey beneficio, Frank Knight (1921), uno de los fundadores de la escuela de Chicago, subrayó la distinción entre riesgo e incertidumbre: el riesgo se puede describir en forma probabilística, la incertidumbre no. Puesto que no existe una sola manera de definir la probabilidad, la noción de incertidumbre puede variar dependiendo de la definición de probabilidad. Este artículo se propone reconstruir las distintas nociones de incertidumbre y responder a la queja de Vickers (19791980), cuando señaló la falta de una explicación adecuada de en qué situaciones se podía hablar de probabilidad y en cuáles no.

Knight formuló la distinción entre riesgo e incertidumbre para explicar los beneficios de un modo intermedio entre el realismo de Marshall y la formalización de J. B. Clark. Los beneficios (el monto que excede al costo en una situación normal o estática) solo se pueden entender cuando hay incertidumbre, puesto que la participación normal de los factores ya incluiría la que obedece a razones probabilísticas. Por tanto, para entender los beneficios se debía separar la noción de incertidumbre de la de riesgo.

Paralelamente, Keynes en su Tratado sobre la probabilidad (1921) expuso la idea de probabilidad como peso lógico de los argumentos, un aspecto subjetivo ligado a la lógica o al proceso de pensamiento del observador, y no como un resultado de cálculos de la distribución

\footnotetext{
* Profesor de la Universidad Nacional y de la Universidad Externado de Colombia, Bogotá, Colombia, [mariogarciamo@gmail.com]. Agradezco los comentarios de Mauricio Pérez y dos evaluadores anónimos. Fecha de recepción: 16-07-2014, fecha de modificación: 11-03-2016, fecha de aceptación: 20-10-2016. Sugerencia de citación: García M., M. "Probabilidad e incertidumbre, una aproximación desde la historia del pensamiento", Revista de Economía Institucional 18, 35, 2016, pp. 101-122. DoI: http://dx.doi.org/10.18601/01245996.v18n35.06.
} 
empírica de las observaciones. No obstante, aceptó que en algunas situaciones, como el juego de dados o la ruleta, se podían calcular las probabilidades de ese modo.

Parece haber cuatro alternativas. O bien a veces no hay probabilidad en absoluto; o no todas las probabilidades pertenecen a un mismo conjunto de magnitudes medibles en términos de una unidad común; o estas medidas siempre existen, pero en muchos casos son, y deben permanecer, desconocidas; o las probabilidades pertenecen a tal conjunto y es posible determinarlas, aunque no siempre podamos hacerlo en la práctica (Keynes 1921, 33).

La primera situación, en la que no existe la probabilidad, era la que Keynes consideraba aplicable a la economía, como confirmó en otros escritos (1937).

Keynes redactó la Teoría general asumiendo la idea de incertidumbre, aunque no la definió de manera explícita. Esto es claro en el capítulo 12, donde subrayó la precariedad de nuestro conocimiento del desempeño de un negocio a varios años y argumentó:

nuestro conocimiento actual no nos da bases suficientes para una expectativa matemática calculada [...] Las decisiones humanas que afectan el futuro [...] no pueden depender de la expectativa matemática estricta, desde el momento que las bases para realizar semejante cálculo no existen (1936, 139 y 148).

Autores posteriores reconocieron la importancia de la noción de incertidumbre. Koopmans advirtió que no se sabía lo suficiente sobre el problema fundamental de la organización: lidiar con la incertidumbre. Weintraub argumentó que la incertidumbre daba color a la decisión. Shackle insistió en la presencia permanente de la incertidumbre y en que en las decisiones acerca del futuro no sabemos qué va a ocurrir; fue más allá que los demás autores y rechazó el uso de la probabilidad donde Keynes lo habría aceptado.

Pero si tales autores aceptaban la incertidumbre como una característica de la realidad social, Vickers (1979-1980) avanzó en su importancia teórica: 1) el uso del término "elección" solo tiene sentido en condiciones de incertidumbre, de otro modo sería simplemente un comportamiento acorde con alguna regla mecánica (p. ej., una regla de optimización); y 2) la incertidumbre afecta la noción de marginalidad porque en situaciones de incertidumbre no se cumplen las funciones necesarias para poder hablar de margen o de elección en el margen. En otras palabras, debemos estudiar la elección bajo incertidumbre, pero ya no se puede hacer análisis "en el margen" y la elección debe explicarse de otra manera.

Aunque entre los economistas postkeynesianos y austriacos continuó la distinción entre riesgo e incertidumbre, prácticamente desapareció de la corriente principal a partir de Tobin (1959), quien usó 
esos términos como equivalentes e hizo depender la preferencia de liquidez de una situación de riesgo. Desde ese momento, la mayoría de los autores los emplean como sinónimos (Rosser, 2001).

El uso generalizado de modelos estocásticos reforzó esta situación. El desarrollo de la econometría se concentró en definiciones de probabilidad que enfatizaban los elementos matemáticos de su construcción, necesarios para un enfoque axiomático, y no las características extrínsecas a la matemática pero importantes para el economista. Más aún, a medida que se desconoció la construcción histórica de la probabilidad, se olvidó que detrás de las fórmulas utilizadas no existía uno sino varios conceptos de probabilidad.

Si hay diferentes conceptos de probabilidad existen diferentes nociones posibles de incertidumbre. El objetivo de este artículo es definir la incertidumbre de manera rigurosa (aunque no unívoca) y mostrar, desde la perspectiva de la historia del pensamiento, su importancia para los problemas de la economía. En la primera sección se muestra brevemente que los problemas relativos al azar no se estudiaban en forma matemática antes del Renacimiento; la segunda sección repasa la construcción histórica del concepto de probabilidad y reconstruye la noción de incertidumbre para cada una de esas concepciones; la tercera sección examina los tratamientos de la incertidumbre (o su ausencia) en la corriente principal y los problemas de cada tratamiento; por último se examina el enfoque de Keynes y cómo puede ayudar a entender los fenómenos de incertidumbre.

\section{AZAR, FORTUNA Y PROBABILIDAD}

La probabilidad es una noción reciente. Aunque los fenómenos aleatorios ya se habían estudiado, la confluencia entre los aspectos epistemológico y aleatorio de la cuestión solo se dio en el siglo XVII, cuando Pascal y Fermat resolvieron algunos problemas de repartición de las ganancias en el juego de dados (aleae en latín). Desde su nacimiento, la noción quedó entonces al riesgo, es decir, a una situación en la que se conocen todos los eventos posibles y la probabilidad asignada a cada uno de ellos. Así fueran difíciles los cálculos y hubiese necesidad de crear fórmulas para plantear claramente el problema, existía bastante conocimiento del proceso.

Esta situación contrasta con la de incertidumbre. Cabría pensar que en el juego de los dados se conocen todos los eventos posibles (las caras del dado). No obstante, la antigua saga de San Olaf cuenta que los reyes de Suecia y Noruega se jugaron una ciudad fronteriza y ganó el de Noruega cuando su dado se partió en dos y señaló si- 
multáneamente el 1 y el 6, es decir, 7 (Ekeland, 1991, 15). Lo que allí sucedió no pertenece al reino del riesgo, pues lo esencial de esa historia es el carácter inesperado y único del evento. Se trata más bien de la fortuna o el destino, que hacen que ocurra lo que debe ocurrir, pese a lo que hagan los hombres y así no lo esperen.

En su Liber de ludo aleae (Libro de los juegos de azar), uno de los primeros trabajos sobre probabilidad, escrito en 1564 pero publicado póstumamente, Gerolamo Cardano incluyó a los dados entre los juegos basados en la fortuna (1663), a la que definió como

una disposición de los asuntos acorde o contraria a la voluntad o a los planes de un hombre; de manera que no importa cómo actúes, el asunto sale bien o mal, o concuerda con los planes humanos o no concuerda [...] tanto si me quedo en casa como si no me quedo, de todas maneras puede resultar muy malo; suceda lo que suceda, estamos sujetos a la autoridad del Príncipe. Así es también en los juegos (ibíd., 48).

Aun para un estudioso de las fórmulas del azar, había un vínculo estrecho entre azar y destino, si bien Cardano también pensaba que la fortuna se podía cambiar por la acción: "la fortuna se cambia mediante algún principio, como se cambia un plan”, por ejemplo ir a la guerra (ibíd., 46, 48).

Christiaan Huygens abordó el problema de la probabilidad en su De ratiociniis in ludo aleae (Sobre los cálculos en los juegos de azar, publicado en 1657), que fue resultado de su visita a París, donde conoció la correspondencia sobre probabilidad entre Pascal y Fermat. Si bien el escrito de Huygens es de apenas 13 páginas, es la primera obra impresa sobre probabilidad. Huygens plantea el problema en términos similares a los de Cardano ("Aunque en los juegos que dependen de la Fortuna el Éxito siempre es incierto, al mismo tiempo es posible determinar de manera exacta, en qué medida es más factible ganar que perder"). La diferencia es que la presencia de la diosa Fortuna solo provoca una ignorancia relativa, pues Huygens calcula la probabilidad de ciertas situaciones particulares ${ }^{1}$.

Aristóteles había clasificado los eventos en tres grupos: eventos ciertos, que ocurren necesariamente; eventos probables, que ocurren la mayoría de las veces; y eventos impredecibles o desconocidos, que ocurren por azar. En la tercera categoría incluyó los resultados de los juegos de azar. Ni los griegos ni los romanos analizaron el problema de los juegos de azar desde una óptica matemática o axiomática, como sí hicieron con la geometría. Quizá por dos razones. La primera es

${ }^{1}$ A diferencia de la mayoría de los autores en la historia de la probabilidad, Huygens no era religioso y estaba más interesado en los fenómenos naturales, a los que consideraba totalmente ajenos a los asuntos religiosos (Bell, 1947). 
que los dados que usaban eran astrágalos (tabas), huesos del pie de ciertos animales y del hombre que tienen cuatro caras, pero que no tienen una estructura uniforme para deducir equiprobabilidades; la segunda es que el uso de dados y del azar en ceremonias religiosas y de predicción mágica - no solo en la antigüedad occidental, sino también en numerosas sociedades antiguas (David, 1962, 35-42)-, podía impedir un abordaje puramente racional.

Cuando los escolásticos funden la visión de Aristóteles y la doctrina cristiana, los eventos impredecibles o desconocidos para el hombre se consideran preestablecidos por Dios. Al discutir la probabilidad, Tomás de Aquino distingue entre conocimiento científico o con certeza, y accidental o azaroso. La probabilidad es aquí la calificación de una proposición, si bien su "peso" ha sido interpretado a veces como una versión primitiva de la frecuencia (Hald, 2003, 30). No obstante, se puede afirmar que el resultado del vínculo entre fortuna y azar en estos casos de la antigüedad y la Edad Media es que, cuando se estudia la probabilidad, el examen es cualitativo y no cuantitativo. Pero no hay que olvidar que la diosa Fortuna, que sobrevivió en el imaginario medieval como imagen de lo inesperado, lo inestable y lo impredecible, también se representaba asociada a los dados (González, 2006, 39) ${ }^{2}$.

La doctrina probabilista es otro ejemplo de enfoque desde un punto de vista religioso. Los probabilistas se concentraron en situaciones prácticas en las que no había certeza, en lo que Aquino habría llamado conocimiento accidental. El mejor ejemplo es el de un sacerdote (no un teólogo) que intenta aconsejar a un fiel para hacer lo correcto en una situación concreta. La dificultad radica en que en esta clase de situaciones suele haber argumentos a favor y en contra de cada alternativa.

Probabilistas como Juan Caramuel expusieron teorías que tomaban en cuenta factores como qué tan bueno era el argumento o si uno o más padres de la Iglesia lo habían defendido (Castrillón, 2003). Propusieron doctrinas que defendían el libre albedrío en situaciones en que había razones en favor de una acción aunque no fueran las más fuertes. Caramuel, que fue inicialmente escolástico y a quien le gustaba defender posiciones polémicas, postuló que una acción era buena si era defendida al menos por una autoridad. Esa posición fue rechazada porque, así, siempre se podía defender cualquier acción.

\footnotetext{
${ }^{2}$ La discusión sobre la fortuna en autores como Maquiavelo (El príncipe, cap. 25) gira alrededor de la idea de que así la fortuna controle la mitad de las acciones humanas, es posible gobernar la otra mitad. Esta idea es afín a la función de la polis griega, servir de protección o control del azar-fortuna, aunque esa misión política fuese como la de un barco en altamar (Nussbaum, 1986, 58).
} 
Recordemos que la expresión sic et non se aplicaba a la Biblia porque siempre se podían encontrar argumentos a favor y en contra de la misma cosa leyendo distintas partes del libro.

Aunque el probabilismo fue rechazado por Pascal en sus Cartas provinciales (1657), dejó legados importantes para nuestro tema. Por un lado, mostró la importancia práctica de las situaciones en que no hay certeza completa, más allá de los juegos de azar. Keynes retomará la idea del peso de los argumentos y será uno de los pocos autores modernos que menciona a Caramuel. Por otro lado, el Kibeia de Caramuel y el segundo tratado sobre las probabilidades (1670), incluido en su Mathesis biceps, servirán de antecedente inmediato e inspiración a Pascal para construir su noción de probabilidad.

El desarrollo de la probabilidad en forma cuantitativa no eliminará de inmediato sino que modificará por un tiempo los argumentos que mezclan probabilidad y teología. Como muestra lo que es tal vez el primer ejemplo de teoría de la decisión (fuera del estudio de los juegos de azar): la apuesta pascaliana. Con ella no se intenta demostrar la existencia de Dios, sino mostrar en cambio que, dada la incertidumbre acerca de su existencia y los pagos posibles por creer o no, la decisión racional es creer (Mora, 1989, 139-152).

La conexión entre probabilidad y preocupación religiosa también se encuentra en Bayes y Bernoulli (ambos religiosos). Otro ejemplo de la permanencia del vínculo conceptual con lo divino en un estudioso de las leyes del azar es el de John Arbuthnot (1710), para quien "el exacto equilibrio que se mantiene entre el número de hombres y mujeres [...] no es efecto del azar, sino de la Divina Providencia”.

\section{CONCEPTOS DE PROBABILIDAD}

Durante los cuatro siglos en que se ha mencionado la probabilidad, no se ha hecho referencia a un solo concepto, sino a varios.

El enfoque cuantitativo cobra fuerza en los siglos XVI y XVII con el uso cada vez mayor de la razón en el examen del azar. Pero el paso al cálculo numérico de las probabilidades fue bastante rápido y dependió de un concepto en apariencia muy sencillo: la igualdad de probabilidades. Cardano estableció, como "principio principalísimo, la igualdad: de los contrincantes, [...] del lugar, del cubilete, del juego mismo. Y en la medida en que te apartes de esa cualidad, si es en tu contra, eres un tonto, si es a tu favor, eres injusto" $(1663,25)$. Y usó ese principio para calcular las probabilidades de un lance de dados, suponiendo que no están adulterados (ibíd., 30 y ss.). En términos modernos, Cardano asumió equiprobabilidad para las caras del dado. 
Este supuesto se basa en que sería irracional e injusto que se jugara con un dado cuyas caras no fueran equiprobables. Luego será usado por Galileo (Mora, 1989, 64), Pascal (Basulto y Camúñez, 2007, 75) y Bernoulli (Hald, 2003, 113).

El concepto clásico de equiprobabilidad parte de una serie de eventos posibles (cada cara del dado) y de suponer que, si no hay razones para pensar que uno de los eventos sea más frecuente que otro, todos deben ser equiprobables, es decir, deben tener la misma probabilidad. De allí se deduce que la probabilidad de cada una de las caras de un dado perfecto es de $1 / 6$, la de las caras de una moneda, de $1 / 2$, etc. La probabilidad de eventos compuestos (sacar 7 tirando dos dados) se calcula a partir de todas las combinaciones posibles de los eventos primarios. Esta noción de probabilidad parte del conocimiento de todos los eventos posibles.

El enfoque de equiprobabilidad no requiere la observación, o al menos la observación prolongada del fenómeno, sino su comprensión lógica, en particular, el conocimiento de los posibles eventos. Dar mayor importancia a la observación llevaría a un concepto distinto.

El siglo siguiente fue testigo de un cambio de énfasis en el enfoque. E1 aporte más conocido, de Jacob Bernoulli (1654-1705), es lo que hoy se conoce como ley de los grandes números, según la cual, para ensayos independientes de un proceso aleatorio, dados un intervalo y el nivel de seguridad que deseemos del valor de la probabilidad, existe un número tal que, si se repiten los ensayos al menos ese número de veces, el valor promedio de la frecuencia obtenida estará cerca de la verdadera probabilidad dentro del intervalo, con la seguridad deseada. En otras palabras, suponiendo que se conoce la probabilidad, al aumentar el tamaño de la muestra la frecuencia observada tiende a la probabilidad (Tabak, 2004, 34-36).

Bernoulli también estaba interesado en el problema inverso, aunque no lo resolvió. Si no se conoce la probabilidad de un evento, pero sí la frecuencia relativa después de cierto número de ensayos, ¿se puede estimar la probabilidad del evento? Lo importante es que Bernoulli logró mostrar una conexión entre probabilidad y frecuencia observada en procesos aleatorios independientes.

E1 papel de la observación cobró más fuerza con la influencia del empirismo. Entre finales de la década de 1830 y comienzos de la de 1840 , cuatro autores construyen interpretaciones de la probabilidad que avanzan en dirección del enfoque frecuentista. Jakob Friedrich Fries en Alemania, Antoine Augustin Cournot en Francia y Richard Leslie Ellis y John Stuart Mill en Inglaterra. 
Mill estaba interesado en lo que se conocía como probabilidad de las causas: cómo distinguir entre coincidencias casuales y aquellas que son producto de una ley (Mill, 1974, 1148). Mill consideraba que las probabilidades solo se podían establecer en la medida en que tuvieran una base empírica, en que se basaran en "conocimiento producto de la experiencia acerca de la proporción entre los casos en que el hecho ocurre y aquellos en los que no. Todo cálculo de posibilidades se basa en una inducción" (ibíd., 1142).

La teoría de Mill es una crítica a Laplace (cuya concepción examinamos más adelante), pero fue moderando sus afirmaciones en el curso del tiempo. Un cuarto de siglo después, el religioso, matemático y profesor de ciencias morales en Cambridge, John Venn (1834-1923), fue más allá y definió la probabilidad en términos de frecuencias. Más exactamente, definió la probabilidad de un evento como su frecuencia de largo plazo (Tabak, 2004, 97). La definición de Venn fue corregida por el físico y matemático nacido en Lemberg Richard von Mises ${ }^{3}$ (1883-1953), quien incluyó el supuesto de que los resultados futuros no se pueden predecir totalmente a partir de los pasados, es decir, que se trataba de una variable aleatoria (Tabak, 2004, 98).

$\mathrm{E} 1$ enfoque frecuentista fue un intento de fundamentar la probabilidad sobre una base más objetiva que la de la doctrina bayesiana. Suponía que los datos disponibles son una muestra aleatoria de puntos de un conjunto mayor de resultados bien definidos (ibíd., 99), un supuesto que guarda similitud con la idea de Pearson (1857-1936) (egresado de Cambridge e influido por Marx) de que los puntos observados son un subconjunto de otro mucho mayor que tiene el patrón verdadero que se está buscando (Tabak, 2004, 138). Pero en general el investigador se ve reducido a estudiar solo una familia de curvas posibles.

Con espíritu frecuentista, algunos estudiosos decidieron hacer pruebas empíricas para confirmar las probabilidades de tirar una moneda o lanzar dados. Pearson dedicó sus vacaciones a lanzar una moneda 25.000 veces y Weldon realizó 25.000 lanzamientos de 12 dados. Los resultados no parecían seguir las mismas leyes. Se veían afectados por elementos tales como el desgaste de las esquinas de los dados, la falta de uniformidad de las caras (por tener grabados los huecos), etc. (Pearson, 1924, 405). Pero si estos problemas aparecían en elementos físicos relativamente homogéneos, ¿cabría esperar que las mediciones experimentales dijeran algo acerca de las probabilidades conceptuales en situaciones más complejas como las sociales? Esa

\footnotetext{
${ }^{3}$ Hermano menor de Ludwig, el economista, también nacido en Lemberg, en la actual Ucrania.
} 
fue una de las fuentes del escepticismo del joven Keynes (Dostaller, 2005, 111).

Mientras que el enfoque frecuentista parte de la observación de las variaciones, una alternativa importante se basa en el determinismo. Según Laplace, en sus conferencias de 1795, publicadas como Ensayo filosófico sobre las probabilidades,

hemos de considerar el estado actual del universo como el efecto de su estado anterior y como la causa del que ha de seguirle. Una inteligencia que en un momento determinado conociera todas las fuerzas que animan a la naturaleza, así como la situación respectiva de los seres que la componen, si además fuera lo suficientemente amplia como para someter a análisis tales datos, podría abarcar en una sola fórmula los movimientos de los cuerpos más grandes del universo y los del átomo más ligero; nada le resultaría incierto y tanto el futuro como el pasado estarían presentes ante sus ojos (Laplace, 1995, 25).

Aunque en este conocido pasaje emplea las palabras "causa" " "efecto", el determinismo por el que aboga no depende de la noción de causalidad sino de la de predictibilidad. De que cada descripción exhaustiva de la naturaleza en cierto momento, esté unívocamente ligada a la descripción en un momento anterior (Earman, 1986, 6). No se trata de predictibilidad por parte de cualquiera, sino por parte del llamado daimon de Laplace. Si este ser imaginario tuviese el conocimiento de la situación actual, y la capacidad de cálculo apropiada, podría predecir la situación futura. En otras palabras, la predictibilidad de Laplace haría que el determinismo sea una característica del mundo, es decir, una característica ontológica.

En contraste con el determinismo a nivel ontológico, Laplace señala que la probabilidad surge porque no se conocen los mecanismos exactos.

La regularidad que la astronomía nos muestra en el movimiento de los cometas tiene lugar, sin ningún género de dudas, en todos los fenómenos. La curva descrita por una simple molécula de aire o de vapor está determinada de una forma tan exacta como las órbitas de los planetas. Entre ellas no hay más diferencia que la derivada de nuestra ignorancia. La probabilidad es relativa en parte a esta ignorancia y en parte a nuestros conocimientos (Laplace, 1995, 27).

En otras palabras, la probabilidad es de índole epistemológica, fruto de la ignorancia del investigador. Laplace da el ejemplo de tres urnas, dos con bolas blancas y una con bolas negras. ¿Cuál es la probabilidad de que, al sacar una bola de la urna c, sea negra? Sin más información, la probabilidad sería de $1 / 3$ porque no hay razones para pensar que la probabilidad sea mayor para А о para в. Hasta aquí parecería ser un argumento de equiprobabilidad. Pero si sabemos que la urna A tiene bolas blancas, el argumento de equiprobabilidad lleva a asignar una 
probabilidad de $1 / 2$ a que la bola de la urna c sea negra (ibíd., 29). La probabilidad cambió no porque haya cambiado el mundo sino porque cambió la información disponible. Si bien aquí no hay espacio para considerar los cuidadosos matices de la exposición de Laplace en sus escritos, la forma como se entendía a este autor a comienzos del XIX (p. ej., por de Morgan) era la reducción de la probabilidad a lo que hoy llamaríamos creencia racional. Es posible entender a Laplace como parte del intento de utilizar la probabilidad basada en el grado de creencia en la construcción de una lógica probabilística de la inferencia científica, que también incluiría Los principios de las ciencias de Jevons, el Tratado sobre la probabilidad de Keynes, Verdad y probabilidad de Ramsey y Fundamentos lógicos de la probabilidad de Carnap (Strong, 1978).

En contraste con el enfoque frecuentista, el bayesiano (basado en las ideas del clérigo presbiteriano Thomas Bayes y, sobre todo, en las de Laplace) toma en cuenta situaciones en las que la frecuencia no es la base de la probabilidad. Si se parte de un grupo de hipótesis mutuamente excluyentes, de modo que una y solo una puede ser cierta (Tabak, 2004, 97), con la información disponible se pueden asignar números a las diferentes hipótesis y actualizarlos a medida que se obtiene más información. Esta especie de enfoque marginalista parte de probabilidades iniciales arbitrarias (subjetivas) que se van mejorando de tal modo que se puede elegir la hipótesis más plausible.

En la primera edición de su Sistema de lógica, Mill critica duramente a Laplace por no tomar en cuenta la observación.

$\mathrm{Si}$ su dominio indiscutido de los instrumentos matemáticos para el cálculo de los resultados de unos datos dados implicara necesariamente un juicio igualmente correcto de lo que dirían los datos, difícilmente expresaría mi convicción de que, en esta opinión, [Laplace] se ha equivocado completamente; que su fundamento es del todo insuficiente para la superestructura que en él se basa; y que todo cálculo racional de probabilidades de eventos conlleva implícita una condición esencial, que la afirmación de Laplace subestima o indica de manera tan vaga que el lector no la nota, ni el autor tiene en cuenta (Mill, 1974, 1140-1141).

Esta afirmación aparece en la primera edición (1843) pero desapareció a partir de 1846 por los comentarios que le hizo el astrónomo Herschel (Strong, 1978).

El enfoque bayesiano fue criticado porque el grado de plausibilidad de una hipótesis no era, a los ojos de muchos matemáticos, una base sólida para una matemática rigurosa, menos aún cuando el cálculo de probabilidades dependía de decisiones subjetivas del investigador (ibíd., 98). Aunque desplazado por el frecuentista, el enfoque bayesiano revivió en 1939 con la publicación de Theory of probability de 
Harold Jeffreys, profesor de Cambridge (Saint John's), que mostró la manera de aplicarlo en situaciones que se abordaban con el enfoque frecuentista (ibíd., 100). Otro autor que adoptó la posición bayesiana fue Ramsey, que criticó las ideas de Keynes desde un punto de vista bayesiano (Ramsey, 1926).

Con el trabajo de Savage la idea de probabilidad subjetiva cobra mayor importancia. No hace falta conocer las probabilidades reales. Para explicar el comportamiento humano basta conocer las probabilidades subjetivas de cada individuo; la teoría de la decisión explicará el resto.

\section{CONCEPTOS DE INCERTIDUMBRE}

Como se mostró en la sección anterior, no hay una sola definición de probabilidad. Si la incertidumbre es una situación no probabilística, existen diversas definiciones posibles, según la noción de probabilidad de la que se parta. La tarea de esta sección es reconstruir la definición de incertidumbre adaptada a cada caso.

Es posible definir la incertidumbre en términos de la noción clásica de probabilidad como una situación en la que no se conocen todos los eventos posibles y no es claro cuáles son los eventos equiprobables. En el desempeño de una empresa nueva, por ejemplo, ¿cuáles son los eventos equiprobables? ¿Quebrar o mantenerse? En ese caso el argumento de igual probabilidad asignaría un valor de $1 / 2$ a cada evento. ¿Tener ganancias altas, medias o bajas? Las probabilidades serían de $1 / 3$ cada una, pero los términos "altas" o "bajas" son ambiguos. ¿Tener ganancias de $\$-1$ millón, $\$ 0, \$ 1$ millón, $\$ 2$ millones, y así sucesivamente hasta $\$ 20$ millones? En ese caso la probabilidad de cada evento sería de $1 / 22$; pero, ¿por qué parar en $\$ 20$ millones? Se podría parar en 40 y las probabilidades serían de $1 / 42$. A diferencia del lanzamiento de un dado, en una situación como esta no se pueden listar todos los eventos posibles y, por tanto, no es posible usar la equiprobabilidad para asignarles valores iguales a todos.

La dificultad es que en algunas situaciones no se pueden definir los eventos, no solo porque pueden ser infinitos, sino porque ni siquiera podemos imaginarlos de antemano (como suele ocurrir en política y como ocurrió en el caso del dado partido ya mencionado). La infinitud de los eventos no es un problema per se, Bernoulli aplicó la equiprobabilidad a infinidad de eventos para calcular la esperanza de vida calculando el límite cuando el número de intervalos temporales tendía a infinito (Hald, 2003, 113). El problema aparece cuando muchos de esos infinitos eventos posibles son insospechados. 
En el contexto frecuentista la incertidumbre se podría definir como una situación para la que no hay observaciones previas (o se tienen muy pocas) y, por tanto, no se conocen las frecuencias. En otras palabras, el enfoque frecuentista depende de la repetición del experimento, pero muchas situaciones de la vida social y económica son únicas y no hay repetición de los eventos. Consideremos, por ejemplo, la probabilidad de que un país invada a Rusia en invierno. Hay dos ejemplos (las campañas de Napoleón y de Hitler) de un ejército invasor que perdió contra el general invierno. ¿Cuál es la probabilidad de que un nuevo invasor tenga éxito allí donde otros dos fracasaron? Esta es una situación de incertidumbre en la que desde el punto de vista frecuentista no tendría sentido hablar de probabilidad.

$\mathrm{El}$ artificio de Laplace para definir el determinismo ontológico no es satisfactorio porque no sabemos qué características son admisibles para el daimon de Laplace. ¿Su capacidad de cálculo es superior a la de una máquina de Turing? Si aceptamos la sugerencia de Popper de entender el daimon no como una especie de dios sino como un súper científico con capacidad limitada para plantear los números que, por tanto, tiene un margen de error, una situación como la del caos determinístico (Baumol y Benhabib, 1989) no sería determinística en el sentido de Laplace. El punto es que la ignorancia deja de ser una característica de los simples mortales y es también un problema del ente imaginario con el que describimos el universo. En otras palabras, para Laplace la probabilidad es epistemológica, no ontológica. Pero si se toma en serio su instrumento ontológico, el daimon de Laplace, la ignorancia se convierte en una característica del mundo, no de sus observadores. Por tanto, la ignorancia, que en el enfoque bayesiano es de carácter epistemológico, al mirarla más de cerca es de carácter ontológico.

Si se omiten esas contradicciones internas, el enfoque bayesiano parece hacer irrelevante la idea de incertidumbre. Pero en realidad no resuelve el problema, pues supone que los individuos son bayesianos, y hay evidencia experimental abrumadora que muestra que los seres humanos no son bayesianos cuando actúan y cometen toda clase de errores incompatibles con las leyes de la estadística (Kahneman et al., 1982). Para que haya incertidumbre basta que no se conozcan las probabilidades y que los individuos no sean bayesianos.

Para cada una de las tres definiciones de probabilidad tenemos una definición de incertidumbre. Podemos pasar ahora a considerar este problema desde el punto de vista de la economía. 


\section{RIESGO E INCERTIDUMBRE EN LA ECONOMÍA MODERNA}

A diferencia del juego de dados, la mayoría de las situaciones que enfrentamos en la vida cotidiana y económica son de incertidumbre. ¿Cómo entendieron los economistas la idea de incertidumbre?

Una manera fue la de Keynes, quien en $A$ treatise on probability se basó en la teoría de la probabilidad en su versión intuicionista. Allí se concentra en el peso de los argumentos ${ }^{4}$ y en la relación lógica entre los argumentos y el suceso esperado, más que en asignar frecuencias que solo tienen sentido cuando hablamos de situaciones controladas. Estudiar la probabilidad significa entonces examinar cuándo es correcto hablar de posibilidad de un suceso.

Partiendo de la existencia de incertidumbre, los autores postkeynesianos se preguntaron si la incertidumbre es una característica del mundo o de nuestra manera de conocerlo. Es posible que el mundo no asigne probabilidades a los eventos, en cuyo caso la incertidumbre sería de carácter ontológico, es decir, una propiedad de la naturaleza. También es posible que la incertidumbre solo se refiera a las limitaciones del conocimiento, en cuyo caso la incertidumbre es de carácter epistemológico. Autores postkeynesianos como Davidson, Shackle y Kregel se centran en la incertidumbre ontológica (Davidson, 1974), mientras que la epistemológica es un tema más cercano a los trabajos sobre racionalidad acotada de Herbert Simon.

No obstante, la mayoría de los economistas siguió usando modelos de riesgo y no de incertidumbre. Permanecieron así en una tradición determinista proveniente del siglo XVII, según la cual un evento no conduce necesariamente a otro (determinismo estático), pero sí conduce a él con cierta probabilidad (determinismo con azar).

En su renuencia a usar la incertidumbre, la corriente principal de la economía presenta otro punto de continuidad con respecto al papel de la razón y la racionalidad. El uso de la razón es una herencia escolástica, pero la primacía de las matemáticas y la creencia en que la razón está entera en cada uno de los hombres (las divergencias provienen únicamente del método) son un elemento clave en Descartes y otros ilustrados (Schouls, 1982, 23). El argumento pascaliano de la existencia de Dios utiliza un instrumento matemático, el cálculo de probabilidades, que se obtiene del juego de dados y es, por tanto, asequible en principio para cualquier jugador.

\footnotetext{
${ }^{4}$ Peirce discutió en 1878 la noción de peso del argumento y al parecer Keynes no conocía ese escrito (O’Donnell, 1991). Es posible que resucitara la noción de Caramuel y Tomás de Aquino ya mencionada, y que usara argumentos religiosos para defender una manera de pensar agnóstica; el tipo de chiste erudito que le encantaba.
} 
Esta es la visión del mundo que se conserva en la moderna teoría de la decisión. E1 hombre conoce la probabilidad de cada evento y actúa de conformidad. Claro está que ante el argumento de la ignorancia o la inexistencia de probabilidades objetivas es posible que cada individuo asigne las probabilidades que considere ciertas y luego actúe conforme a esas probabilidades subjetivas. Tal fue el aporte de Savage y la base de la teoría de la utilidad subjetiva esperada. No obstante, esta teoría está limitada, en el mejor de los casos, a ser de carácter normativo porque la evidencia empírica apunta nítidamente en su contra (Kahneman et al., 1982; Anand, 1993).

La vía que siguió la teoría de equilibrio general también tiene dificultades. Debreu (1959) formalizó la incertidumbre mediante un árbol que señala los posibles estados de naturaleza, para cada uno de los cuales deberían existir mercados contingentes. Aquí, el problema no es tanto valorar la opción de cada uno de esos estados, sino que la incertidumbre implica la existencia de innumerables estados que el individuo ni siquiera puede imaginar. Aun si se los conociese, no es claro que la capacidad humana de razonamiento permita hacer todos los cálculos necesarios, así como no podemos calcular todas las jugadas posibles en ajedrez, aunque sean finitas.

Las expectativas racionales suponen que los agentes conocen el modelo verdadero, aunque no nos dicen cómo hacen para coincidir en uno de los múltiples equilibrios de tales modelos. Y el problema no para allí. Consideremos un modelo caótico determinista (Baumol y Benhabib, 1989); aun si se conoce el modelo subyacente, los errores de cálculo (p. ej., una aproximación al cuarto decimal por computador) crecen exponencialmente de modo que es prácticamente imposible la predicción. De nada sirve conocer el modelo porque persiste la incertidumbre sobre el futuro ${ }^{5}$.

Una aproximación a la idea de incertidumbre desde otro ángulo son los modelos de varianza infinita propuestos por Mandelbrot. Estos modelos fueron rechazados por la comunidad de finanzas y economía porque no encajaban fácilmente en la idea de equilibrio; por ello, el origen de la econofísica estuvo asociado al trabajo de los físicos y no al de los economistas (Salazar, 2016). Pero la incertidumbre va incluso más allá de las distribuciones de Pareto-Levy que usa Mandelbrot. Un modelo que se construye a partir de relaciones observadas supone en esencia que el futuro es como el pasado. Si en el periodo de observación hubo precios crecientes, el modelo supone de manera implícita

\footnotetext{
5 Este es el significado del signo del exponente de Liapunov en un modelo caótico.
} 
que los precios seguirán creciendo, como ocurrió con los modelos de valoración que eventualmente llevaron a la crisis subprime (Pérez, 2007). Se podría argumentar que el problema se resuelve alargando el periodo de observación o utilizando modelos no gaussianos. Pero el problema de fondo es que siempre pueden ocurrir eventos nuevos. La historia muestra la importancia de la novedad, pero no permite decir cuál será la próxima novedad.

Desde Tobin (1959), los trabajos sobre mercado de capitales hacen énfasis en la actitud frente al riesgo pero olvidan que en general los individuos también suelen sentir aversión a la incertidumbre ${ }^{6}$. En un experimento famoso realizado por Ellsberg $(1961)^{7}$, se pidió hacer apuestas sobre una urna que tenía 50 bolas rojas y 50 negras y otra que contenía 100 bolas rojas y negras sin conocer la proporción. Las apuestas mostraron que los individuos preferían apostar a que saliera una bola roja en la primera urna, más que apostar a que saliera una bola roja en la segunda urna. Es decir, los individuos son aversos a la incertidumbre y prefieren una situación de mal riesgo a una que no conocen: "más vale malo conocido que bueno por conocer".

Cabe señalar que el ejercicio de Ellsberg no pretendía ser un experimento científico controlado. Fue un ejercicio mental que respondieron economistas importantes de la época, incluidos Samuelson, Debreu y Savage (¡las respuestas de este último no se ajustaban a su propia teoría!).

Años después, a comienzos de los setenta, Ellsberg protagonizó un caso que se puede explicar precisamente por su paradoja. Como consultor de la Rand Corporation para el Departamento de Defensa de Estados Unidos, tuvo acceso a información secreta que mostraba que desde hacía varias administraciones, para el gobierno estadounidense era claro que no podía ganar la guerra de Vietnam; sin embargo los sucesivos gobiernos habían preferido continuar la guerra con un absurdo costo en vidas humanas. Para el gobierno, la opción era un resultado malo seguro (continuar una guerra eventualmente perdida) o una situación sin precedentes en caso de retirar sus tropas (Sheinkin, $2015)^{8}$. Los gobiernos prefirieron el mal resultado. El punto es que la aversión a la incertidumbre (o ambigüedad, como la llama Ellsberg) es importante en la vida real, no una curiosidad teórica.

\footnotetext{
${ }^{6}$ Ellsberg estudia una situación de incertidumbre, pero prefiere referirse a ella como ambigüedad señalando que el jugador no conoce la probabilidad aunque esté definida.

7 Ejercicios similares fueron propuestos por Keynes (1921, 75-76) y Knight (1921, 219).

${ }^{8}$ Ellsberg reveló los documentos, conocidos como "los papeles del Pentágono", al New York Times y al Washington Post en 1971.
} 
Una vía distinta fue propuesta por Gillies (2006), inspirado en Keynes. Gillies subraya el carácter social del comportamiento humano y señala que las creencias se tornan homogéneas al interior del grupo. A partir de allí sugiere construir una teoría de la probabilidad intersubjetiva, definida como los cocientes de apuestas que acuerden adoptar los miembros del grupo. Esta teoría evita fenómenos de libro holandés (una secuencia de apuestas que dé como resultado ganancias con el $100 \%$ de probabilidad). E1 problema es que la teoría no solo supone racionalidad individual sino acuerdos racionales explícitos dentro de los grupos sociales, cuando en realidad operan muchos elementos no conscientes, producto de reglas, hábitos, en fin, de instituciones (Hodgson, 1997) o producto de sesgos cognitivos, y esos elementos intervienen incluso en grupos de individuos presumiblemente racionales, como el de los académicos en la construcción de paradigmas (García y Chicaíza, 2014).

\section{INCERTIDUMBRE Y ESPÍRITUS ANIMALES 9}

Keynes usó varios métodos para tratar las expectativas de corto y largo plazo en condiciones de incertidumbre (Kregel, 1976). E1 primero supone que las expectativas de corto y largo plazo se cumplen y no se contradicen entre sí (modelo de equilibrio estático); el segundo modelo supone que las expectativas de corto plazo pueden no cumplirse pero no afectan a las de largo plazo (modelo de equilibrio estacionario), y el tercero supone que el incumplimiento de las expectativas de corto plazo afecta a las de largo plazo (modelo de equilibrio cambiante).

La dinámica de los tres modelos puede ilustrarse de la siguiente manera. Las expectativas de largo plazo están representadas por un ciclista; las de corto plazo, por un perro. En el modelo de equilibrio estático el ciclista lleva al perro en la canasta de la bicicleta; en el de equilibrio estable, el perro va siguiendo a la bicicleta y se puede acercar o alejar sin que el ciclista cambie su rumbo; en el de equilibrio cambiante, el ciclista intenta acercase al perro cuando este se aleja demasiado y las dos rutas se afectan mutuamente. En este último el tiempo importa y el mundo es no ergódico, es decir, no se puede reducir, con manipulaciones algebraicas, a una serie con media constante. De ahí que algunos postkeynesianos, de Joan Robinson a Paul

9 Aquí no se pretende presentar la teoría de Keynes sobre la probabilidad sino el tratamiento de la incertidumbre en sus textos económicos finales. Sus conocimientos sobre probabilidad eran extensos y al parecer incluían áreas poco conocidas de la escolástica española (Castrillón, 2003). Baste señalar que su enfoque original era intuicionista; en los años treinta no dedicó mucho tiempo al trabajo teórico sobre probabilidad, que data de una década anterior. 
Davidson, consideren que la tendencia de largo plazo no exista más que en la mente de las personas y sea en realidad una mera sucesión de cortos plazos.

Al estudiar las decisiones de inversión, que dependen de las expectativas de largo plazo, cada instante es único e irrepetible y constituye un punto de ruptura y discontinuidad. Por ejemplo, la decisión de ampliar la planta en un momento se enfrenta con la situación actual de los competidores, de la demanda y de la tecnología, es decir, se enfrenta con una conjunción de elementos que no ha ocurrido antes y que jamás se volverá a presentar. En tal caso, las decisiones son irreversibles y lo que suceda en el corto plazo afectará las expectativas acerca del largo plazo. El modelo de equilibrio cambiante incorpora, pues, una de las características olvidadas por la teoría neoclásica: la irreversibilidad del tiempo. Como la situación es única, simplemente no existe una base para calcular las probabilidades y el individuo, para actuar, debe enfrentar una situación en la que la razón no le da asidero para decidir y una vez actúa es demasiado tarde para corregir el curso. Este es uno de los casos en que Descartes - para quien la razón debía primar sobre la voluntad-consideraba que la voluntad impulsaba al error y al pecado al ir más allá de donde la razón permite ver claramente.

Keynes (1937) considera que, ante la incertidumbre, el individuo basa su decisión en convenciones. La persona supone, sin nada que se lo garantice, que mañana el mundo será como ha sido hasta hoy y que el sol saldrá nuevamente. La convención le permite cerrar la discontinuidad creada por la irreversibilidad del tiempo. Cuando en el mercado de valores alguien toma una decisión basándose en un diagrama de precios de los días anteriores y una fórmula que le dice cómo cambiarán (lo que se suele llamar análisis técnico), está usando una convención en una situación en la que la razón no lo llevaría muy lejos.

De la misma manera, Keynes señala que la inversión no depende de la razón sino de los espíritus animales (animal spirits) de los inversionistas, "de un resorte espontáneo que impulsa a la acción de preferencia que a la quietud" (Keynes, 1936, 147). "Es probable que el promedio real de los resultados de las inversiones, aún en periodos de progreso y prosperidad, haya defraudado las esperanzas que indujeron a invertir" (ibíd., 137). Aunque sean benéficas para la actividad económica, tales inversiones no fueron producto de la razón, y hay que buscar su causa en otro lado. "Es nuestra inclinación natural a la actividad la que hace girar las ruedas escogiendo nuestro ser racional 
entre las diversas alternativas lo mejor que puede, calculando cuando hay oportunidad, pero con frecuencia hallando el motivo en el capricho, el sentimentalismo o el azar" (ibíd., 148).

Keynes condensó estos elementos mediante la expresión animal spirits. Los animal spirits aparecen en la literatura médica desde Galeno, para quien el hígado emanaba espíritus naturales que circulaban por las venas; el corazón, espíritus vitales que circulaban por las arterias; y el cerebro, espíritus animales que circulaban por los nervios. Estos últimos eran importantes para explicar la sensación y el movimiento. Descartes intentó integrar la explicación de Galeno y el descubrimiento de Harvey de la circulación de la sangre (Koppl, 1991). Para Descartes los espíritus animales eran minúsculas partículas de la sangre destiladas por el calor del corazón que entraban por los poros del cerebro y circulaban por los nervios para llegar a los músculos causando el movimiento. Los espíritus animales eran afectados por la mente y por las emociones; esta batalla entre cuerpo y alma podía llevar a juicios falsos cuando las emociones triunfaban, y conducir así al error. Descartes consideraba que las decisiones basadas en opiniones verdaderas nunca daban motivo para arrepentirse o lamentarse, mientras que las basadas en opiniones falsas siempre lo hacían.

En sus estudios de pregrado en Cambridge, Keynes estudió la doctrina de Descartes y en sus apuntes comentó al respecto: "acción mental inconsciente" (Moggridge, 1991). La expresión espíritus animales era entonces una frase muy usada en literatura (Visser, 1991), incluso Marx la utilizó para explicar la mayor eficiencia del trabajo en grupo (Ozawa, 1991). En todo caso, el uso de la expresión por parte de Keynes vuelve cabeza abajo la doctrina de Descartes, puesto que son esos espíritus animales los que permiten que exista la inversión, y sostienen la economía cuando la razón solo conduciría a la inacción y a la crisis por falta de información ${ }^{10}$.

Puesto que los espíritus animales operan de una manera que no es necesariamente consciente, cabe tener en cuenta que Keynes daba importancia a la teoría de Freud, cuyas obras leía a medida que otro miembro del grupo de Bloomsbury, John Strachey, las traducía al inglés (Winslow, 1990). La teoría que aparece en el Tratado sobre el dinero y que se convertiría en la preferencia por liquidez en la Teoría general es claramente freudiana.

\footnotetext{
${ }^{10}$ Keynes gustaba de este tipo de ironía erudita. Ver, p. ej., el uso en el Treatise on money de la jarra de las Danaides (alusión pagana) para mostrar los malos efectos del ahorro; y el uso de la taza de la viuda (alusión a la historia del profeta Elías) para mostrar las virtudes del consumo y la vida disipada.
} 
Al estudiar el funcionamiento de la economía, Keynes no se dedica a buscar un mecanismo - de relojería o de azar- como se haría en las ciencias físicas; sino que toma en cuenta la acción humana (el libre albedrío, si se quiere) que se manifiesta en expectativas, convenciones y espíritus animales.

También quiero subrayar enfáticamente que la economía es una ciencia moral. Ya mencioné que trabaja con la introspección y con valores; también lo hace con motivos, expectativas e incertidumbres psicológicas. Siempre hay que estar en guardia contra el tratamiento del material como si fuera constante y homogéneo. Es como si la caída de la manzana al piso dependiera de los motivos de la manzana, de si vale la pena caer al piso, de si el piso quisiera que la manzana cayera, y de los erróneos cálculos de la manzana acerca de su distancia al centro de la tierra (Keynes, 1937, 300) ${ }^{11}$.

Así, al determinismo del reloj y al determinismo de los dados, Keynes enfrenta la ignorancia que inventa el orden del mundo cuando no lo hay o no se lo conoce. De ahí que el orden mismo sea construido socialmente y esté sujeto a modificación. Construido socialmente porque no basta que la persona siga una convención; para ser tal, la convención debe ser seguida y validada por la colectividad. Sujeto a modificación porque apenas la sociedad siga otro patrón, el orden del mundo cambiará en consecuencia.

\section{CONCLUSIÓN}

La crítica del concepto de probabilidad y el énfasis en la incertidumbre involucran pasos metodológicos más complejos de lo que parece a simple vista. Puesto que no hay un solo concepto de probabilidad, y han existido al menos tres versiones difundidas: la frecuentista, la de equiprobabilidad y la subjetiva, este artículo reconstruye la definición de incertidumbre adaptada a cada caso. Argumenta que la mayor parte de los economistas se ha concentrado en las situaciones de riesgo, dejando a un lado la importancia de la incertidumbre. Muestra que Keynes elaboró una conceptualización que toma en cuenta el carácter social de los humanos y la naturaleza incierta del medio en que se desenvuelven. Las convenciones permiten entender la acción humana bajo estas premisas, sin acudir a supuestos irreales sobre la racionalidad y la capacidad cognitiva de los agentes.

${ }^{11}$ Este no es un rechazo a los estudios empíricos, sino a los hábitos que puede crear la modelación matemática. Keynes señala más adelante: “El constructor de modelos no tendrá éxito a no ser que corrija constantemente sus estimaciones mediante la íntima y desordenada familiaridad con los hechos a los que se aplica su modelo" (ibíd.). 


\section{REFERENCIAS BIBLIOGRÁFICAS}

1. Anand, P. Foundations of rational choice under risk, Oxford, Clarendon, 1993.

2. Arbuthnot, J. "Un argumento en favor de la Divina Providencia, tomado de la constante regularidad observada en los nacimientos de ambos sexos" [1710], reimpreso en M. de Mora, Los inicios de la teoría de la probabilidad, Bilbao, Universidad del País Vasco, 1989, pp. 170-174.

3. Baumol, W. y J. Benhabib. "Chaos: Significance, mechanism, and economic applications", Journal of Economic Perspectives 3, 1, 1989, pp. 77-105.

4. Bell, A. E. Christian Huygens and the development of science in the Seventeenth Century, Londres, Edward Arnold \& Co, 1947.

5. Cardano, G. Libro de los juegos de azar [1663], reimpreso en M. de Mora, Los inicios de la teoría de la probabilidad, Bilbao, Universidad del País Vasco, 1989, pp. 22-57.

6. Castrillón, A. "Probabilismo: ética y economía", Economía Institucional 5, 9, 2003, pp. 55-77.

7. David, F. N. Games, gods and gambling, Nueva York, Hafner, 1962.

8. Davidson, P. Money and the real world, Londres, Palgrave MacMillan, 1972.

9. Debreu, G. Teoria del valor [1959], Barcelona, Antoni Bosch, 1973.

10. Dostaller, G. Keynes et ses combats, París, Albin Michel, 2005.

11. Ekeland, I. Al azar; la suerte, la ciencia y el mundo, Barcelona, Gedisa, 1991.

12. Ellsberg, D. "Risk, ambiguity and the Savage axioms", Quarterly Journal of Economics 75, 4, 1961, pp. 643-669.

13. Gillies, D. "Keynes and probability", R. E. Backhouse y B. W. Bateman, eds., The Cambridge companion to Keynes, Cambridge, Cambridge University Press, 2006, pp. 199-216.

14. García M., M. y L. Chicaíza B. "Las decisiones de los economistas: Coase y los sesgos cognitivos en el trabajo teórico", Revista Economía Institucional 15, 29, 2014, pp. 21-39.

15. González G., J. M. La diosa Fortuna; metamorfosis de una metáfora politica, Madrid, A. Machado Libros, 2006.

16. Hald, A. A History of probability and statistics and their applications before 1750, Hoboken, NJ, John Wiley \& Sons, 2003.

17. Hodgson, G. M. "The ubiquity of habits and rules" [1997], "La ubicuidad de los hábitos y las reglas", Revista de Economía Institucional 2, 3, 2000, pp. 11-43.

18. Huygens, Ch. Hugenii, Cristiani; Libellus de ratiocinis in ludo aleae [1657], The value of all chances medskip in games of fortune; cards, dice, wagers, lotteries, etc. Mathematically demonstrated, Londres, S. Keimer, 1714.

19. Kahneman, D.; P. Slovic y A. Tversky. Judgement under uncertainty: Heuristics and biases, Cambridge, Cambridge University Press, 1982.

20. Keynes, J. M. A treatise on probability, Nueva York, Harper and Row, 1921.

21. Keynes, J. M. Teoría general de la ocupación, el interés y el dinero [1936], México DF, Fondo de Cultura Económica, 1986. 
22. Keynes, J. M. “The general theory of employment”, Quarterly Journal of Economics 51, 2, 1937, pp. 209-223.

23. Knight, F. Risk, uncertainty and profit, Boston, Houghton Mifflin, 1921.

24. Koppl, R. "Retrospectives: Animal spirits", Journal of Economic Perspectives 5, 3, 1991, pp. 203-210.

25. Kregel, J. "Economic methodology in the face of uncertainty: The modeling methods of Keynes and the Post-Keynesians", Economic Journal 86, 346, 1976, pp. 209-225.

26. Laplace. P.-S. Ensayo filosófico sobre las probabilidades [1814], Barcelona, Altaya, 1995.

27. Mill, J. S. A system of logic ratiocinative and inductive, J. M. Robson, ed., Collected works of John Stuart Mill, vols. 7 y 8, Toronto, University of Toronto Press, 1974.

28. Moggridge, D. Maynard Keynes: An economist's biography, Londres, Routledge, 1992.

29. Mora Ch., M. de. Los inicios de la teoría de la probabilidad, Bilbao, Universidad del País Vasco, 1989.

30. Nussbaum, M. The fragility of goodness; luck and ethics in Greek tragedy and philosophy, Cambridge, Cambridge University Press, 1986.

31. O’Donnell, R. M. Keynes: Philosophy, economics and politics, Londres, MacMillan, 1991.

32. Pascal, B. Cartas provinciales [1657], Madrid, Imprenta del Colegio de Sordomudos y Ciegos, 1846.

33. Pearson, K. The life, letters and labours of Francis Galton, vol. 2, Researches of Middle Life, Cambridge, Cambridge University Press, 1924.

34. Pérez S., M. "El origen del pánico de 2008: la crisis del mercado de crédito hipotecario en Estados Unidos”, Economía Institucional 9, 19, 2007, pp. 19-54.

35. Ramsey, F. P. "Truth and probability" [1926], F. P. Ramsey, The foundations of mathematics and other logical essays, Londres, Routledge and Kegan Paul, 1931.

36. Rosser Jr., J. B. “Alternative Keynesian and Post Keynesian perspectives on uncertainty and expectations", Journal of Post Keynesian Economics 23, 4, 2001, pp. 545-566.

37. Salazar T., B. "Mandelbrot, Fama and the emergence of econophysics", Cuadernos de Economía 35, 6, 2016, pp. 637-662.

38. Schouls, P. A. Descartes and the Enlightment, Montreal, McGill-Queens University Press, 1989.

39. Sheinkin, S. Most dangerous: Daniel Ellsberg and the secret history of the Vietnam War, Nueva York, Roaring Brook, 2015.

40. Strong, J. V. "John Stuart Mill, John Hershel and the 'probability of causes", Proceedings of the Biennial Meeting of the Philosophy of Science Association, vol. 1, 1978, pp. 31-41.

41. Tabak, J. Probability and statistics: The science of uncertainty, Nueva York, Facts on File, 2004.

42. Tobin, J. "Liquidity preference and monetary policy", Review of Economic Studies 29, 2, 1947, pp. 124-131.

43. Tobin, J. "Liquidity preference as behavior towards risk", Review of Economic Studies 25, 1959, pp. 65-86. 
44. Vickers, J. “Uncertainty, choice, and the marginal efficiencies”, Journal of Post Keynesian Economics, 2, 2, 1979-1980, pp. 240-254.

45. Winslow, T. "Bloomsbury, Freud and the vulgar passions", Social Research 57, 4, 1990, pp. 785-819. 